\title{
Pediatric duodenal wall hematoma; an unexpected differential of an abdominal cyst
}

\section{Introduction}

Intramural duodenal hematoma (IDH) is a rare and often a missed cause of proximal gastrointestinal tract obstruction. ${ }^{1}$ Mostly, it occurs following blunt abdominal trauma in children, but non-traumatic cases have been implicated including anticoagulant therapy, pancreatitis, bleeding disorders, and upper endoscopy. ${ }^{2,3}$ We present a unique case of duodenal hematoma presented with an abdominal mass and duodenal obstruction in a 4-year-old girl and was misdiagnosed for the more commonly encountered cystic abdominal masses.

\section{Case report}

A previously healthy 4-year-old girl presented to our institute complaining of bilious vomiting and abdominal distension. On general examination, the vital signs were within average range and the patient was fully conscious although there was some dehydration. Abdominal examination revealed moderate distension mostly in the epigastric region with no signs of peritonitis. A palpable abdominal mass about $10 \times 10 \mathrm{~cm}$ with smooth surface and ill-defined edges was noted in the umbilical region.

Laboratory investigations revealed low hemoglobin level $(\mathrm{Hb}=8 \mathrm{gm})$ with mild leukocytosis. Serum electrolytes and coagulation profile were within normal limits. Ultrasound (US) abdomen revealed large intra-peritoneal encysted fluid collection about $8 \times 2 \times 5 \mathrm{~cm}$ medial to the descending colon with multi-layered wall, turbid content and internal septations. This is associated with multiple mesenteric lymphadenopathy. The mass displaces the descending colon laterally and the small bowel loops, which are seen collapsed, to the right side. CT abdomen with IV and oral contrast revealed a large intraabdominal cystic lesion about $4 \times 10 \times 10 \mathrm{~cm}$ abutting aorta, IVC and renal vessels posteriorly denoting a retro-peritoneal origin and displacing the distal duodenum anteriorly and the descending colon postero-lateral. The mass is of inverted $\mathrm{C}$ shape with imperceptible wall and turbid content (Figure 1) (Figure 2). Enteric duplication cyst was considered as a main differential and less likely pseudopancreatic cyst. Serum amylase was ordered and revealed to be within normal range.

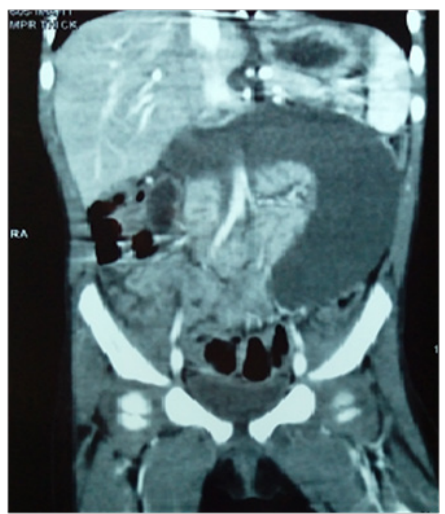

Figure I Coronal CT abdomen revealing inverted C-shape abdominal cystic lesion.
Volume 9 Issue | - 2019

\author{
Ahmed Elrouby,' Mostafa Kotb ${ }^{2}$ \\ 'Lecturer of Pediatric Surgery,Alexandria Faculty of Medicine, \\ Egypt \\ ${ }^{2}$ Assistant Lecturer of Pediatric Surgery, Alexandria Faculty of \\ Medicine, Egypt
}

Correspondence: Mostafa Kotb, Department of Pediatric Surgery, Alexandria Faculty of Medicine, Alexandria, Egypt, Tel 00201062333848,Email mostafa.rashad@alexmed.edu.eg

Received: January 20, 2019 | Published: February 06, 2019

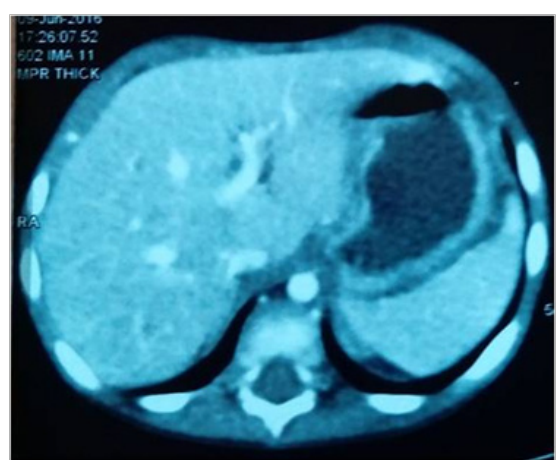

Figure 2 Axial CT abdomen showing imperceptible cyst wall with mildly turbid content.

After stabilization of the general condition; exploration was decided for the intra-abdominal cyst with midline exploratory incision which revealed huge sub-serosal hematoma compressing the $3 \mathrm{rd}$ and 4th parts of the duodenum (grade II hematoma according to the American Association for Surgery of Trauma) without involvement of the surrounding structure (Figure 3). The serosa was opened followed by evacuation of hematoma leaving intact duodenal wall. Nasogastric tube was kept in place. A drain was inserted followed by closure in layers. The girl started oral feeding after 5 days where she tolerated then discharged on the 7th day postoperatively. Postoperative course was uneventful and she is doing well in the follow up visits.

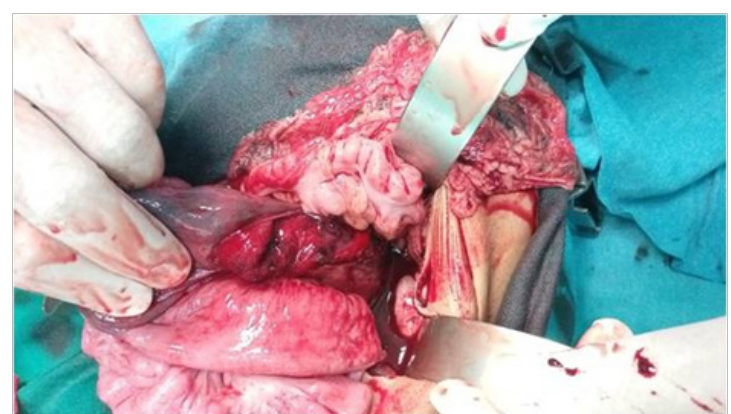

Figure 3 Intra-operative view after evacuation of sub-serosal hematoma of duodenum. 
The parents were asked about history of trauma, where they didn't deny stating the girl was subjected to a blunt trauma while falling from bed two weeks earlier. This pattern of injury seemed weird for us, so the responsible authorities were notified to exclude the possibility of physical child abuse.

\section{Discussion}

Duodenal wall hematoma is a rare condition in children, accounting only $2-3 \%$ cases of blunt abdominal trauma. ${ }^{4}$ It was first described by Mc Lauchlan in $1838 .{ }^{5}$ Owing to its retroperitoneal location, the duodenum is often protected. Most of pediatric duodenal hematomas result from blunt abdominal trauma, contrary to the adults, where penetrating trauma is a more common mechanism. ${ }^{6}$

Other than trauma, non-traumatic causes include anti-coagulant therapy, pancreatitis, vasculitis and malignancy. ${ }^{7}$ Usually the history of trauma isn't elicited except after the diagnosis is established by investigations or even intra-operative. ${ }^{8}$ This is what's happened in our patient in whom the diagnosis of duodenal wall hematoma was only confirmed intraoperative, while the clinical examination and radiological investigations only revealed an abdominal encysted fluid collection without giving a clue to its origin.

Usually after trauma, several days elapse before the onset of symptoms. They vary between vague abdominal pain to proximal intestinal obstruction with tenderness that simulates acute abdomen. ${ }^{9}$ This is explained by the fact that trauma induces contusion and parietal bleeding leading to hematoma formation, that may grows and causes luminal compression. Other infrequent presentations include jaundice induced by mass effect on bile duct, upper abdominal pain, tenderness and elevated pancreatic enzymes denoting that pancreatitis is the eliciting factor. ${ }^{10}$ In our case presented several days after trivial trauma by upper abdominal distension, bilious vomiting with palpable epigastric mass, but the serum amylase level was normal.

For the past decade, there has been a dramatic improvement in the diagnosis and management of duodenal hematoma. It can be diagnosed relatively with ease using computed tomography examination of the abdomen, which typically reveals a thickened bowel wall that often appears as a heterogeneous mass that causes varying degrees of luminal narrowing. ${ }^{11}$ Moreover, the Hounsfield units show characteristic of blood. Typically no further diagnostic studies are needed. ${ }^{11}$

The first line of treatment regimen for IDH is conservative, as spontaneous resolution of the hematoma occurs in most conditions; provided that the patient is stable. ${ }^{12}$ This includes bowel rest, continuous NG decompression, TPN, blood transfusion and correction of coagulopathy if present. Complete resolution of the hematoma with healing of the intestine usually attained within 2 months after diagnosis. ${ }^{12}$ Surgical intervention is only indicated in specific circumstances, for instance if there is significant intraluminal hemorrhage, suspected perforation or doubt in diagnosis as in our case. ${ }^{9,12}$ Surgical evacuation of the hematoma is achieved via laparotomy or more recently, laparoscopy tends to be the best surgical option. ${ }^{13}$ Few authors reported successful outcomes with percutaneous aspiration and endoscopic dilation. ${ }^{14}$ Lastly, bypass surgery (eg. gastrojejunostomy) is reserved for complex cases when there are severe duodenal perforations and/or multiple comorbidities. ${ }^{10}$

Here the possibility of physical child abuse was considered as the pattern of injury is vague. The source of weirdness arises from the fact that being a retroperitoneal structure, duodenum has a degree of protection. ${ }^{15}$ This means that a significant force of trauma is needed in order to cause compression of the duodenum between the vertebral column and a fixed object such as a seatbelt or bicycle handlebar or seatbelt, and hence its injury. ${ }^{16}$ The other possibility is a direct consequence of a direct blow to the epigastrium such as a punch or a kick. ${ }^{15}$ In such cases, both the history and the physical examination can be controversial together with delayed presentation. Once physical child abuse is suspected, complete workup should be done to exclude other injuries. ${ }^{15}$ Moreover, child protection authorities should be notified before discharge. ${ }^{17}$

\section{Conclusion}

Intramural duodenal hematoma is a rare entity especially especially in children and should be considered in differential diagnosis of acquired duodenal obstruction as well as in cystic abdominal swellings.

\section{Acknowledgments}

None.

\section{Conflicts of interest}

The author(s) declared no potential conflicts of interest with respect to the research, authorship, and/or publication of this article.

\section{References}

1. Torba M, Gjata A, Buci S, et al. Delayed presentation of blunt duodenal injuries in children. Case report and review of literature. $G$ Chir. 2013;34(4):122.

2. Hoenisch K, Prommegger R, Schwaighofer H, et al. Intramural duodenal hematoma after upper gastrointestinal endoscopy. Wien Med Wochenschr. 2011;161:441-444.

3. Hou SW, Chen CC, Chen KC, et al. Sonographic diagnosis of spontaneous intramural small bowel hematoma in a case of warfarin overdose. $J$ Clin Ultrasound. 2008;36(6):374-376.

4. Hota D, Rattan KN, Khursheed A, et al. Intramural Duodenal Hematoma In 2-year Old Child. APSP J Case Rep. 2018;9:18.

5. Mc Lauchlan J. Fatal false aneurysmal tumour occupying nearly the whole of the duodenum. Lancet. 1838;2:203-205.

6. Clendenon JN, Meyers RL, Nance ML, et al. Management of duodenal injuries in children. J Pediatr Surg. 2004;39(6):964-968.

7. Chou AL, Tseng KC, Hsieh YH, et al. Intramural duodenal hematoma as a complication of pancreatic cancer. Endoscopy. 2007;39:E107-108.

8. Pandey S, Niranjan A, Mishra S, et al. Retrospective analysis of duodenal injuries: a comprehensive overview. Saudi J Gastroenterol. 2011;17(12):142-144.

9. Abbas MA, Collins JM, Olden KW. Spontaneous intramural smallbowel hematoma: imaging findings and outcome. Am J Roentgenol. 2002;179(6):1389-1394.

10. Ma JK, Ng KK, Poon RT, et al. Pancreatic-induced intramural duodenal haematoma. Asian J Surg. 2008;31(2):83-86.

11. Weil BR, Howard TJ, Zyromski NJ. Spontaneous duodenal hematoma: a rare cause of upper gastrointestinal tract obstruction. Arch Surg. 2008;143(8):794-796.

12. Touloukian RJ. Protocol for the nonoperative treatment of obstructing intramural duodenal hematoma during childhood. Am J Surg. 1983;145(3):330-334. 
13. Simi S, Anoop TM, George KC. Spontaneous intramural duodenal hematoma - a rare cause of upper gastrointestinal obstruction. Am J Emerg Med. 2010;28:642.e1-2.

14. Patterson VM, Goettler CE, Thomas CC. Traumatic duodenal hematoma in a pediatric patient treated by percutaneous drainage. Am Surg. 2011;77:E220
15. Gaines BA, Shultz BS, Morrison K, Ford HR. Duodenal injuries in children: beware of child abuse. J Pediatr Surg. 2004;39(4):600-602.

16. Gaines BA, Ford HR. Abdominal and pelvic trauma in children. Crit Care Med. 2002;30:S416-423.

17. Jain AM. Emergency department evaluation of child abuse. Emerg Med Clin North Am. 1999;17(3):575-593. 